\title{
Role of Diffusion Weighted MRI in Evaluation of Orbital Lesions
}

Ayman Farouk Soliman ${ }^{* 1}$ M.Sc, Mohammad Farouk Aggag ${ }^{1}$ MD, Abdullah EhusseinyAbdelgawwad ${ }^{2}$ MD, Wafik Ebrahim Aly ${ }^{1}$ MD, Ayda Aly Youssef ${ }^{3}$ MD

* Corresponding Author:

Ayman Farouk Soliman

afssmow@yahoo.com

Received for publication January 21, 2020; Accepted May 3, 2020; Published online May 3, 2020.

Copyright 2020 The Authors published by Al-Azhar University, Faculty of Medicine, Cairo, Egypt. All rights reserved. This an openaccess article distributed under the legal terms, where it is permissible to download and share the work provided it is properly cited. The work cannot be changed in any way or used commercially.

doi: $10.21608 /$ aimj.2020.22010.1053

1 Radiodiagnosis Departemnt, Faculty of Medicine -Al-Azhar University

2 Ophthalmology Departemnt, Faculty of Medicine-Al-Azhar University

3 Radiodiagnosis Departemnt, National Cancer Institute -

Cairo University

\begin{abstract}
Background: Orbital lesions represent a spectrum of benign and malignant lesions in adults and children that can be challenging to diagnose and treat. Magnetic resonance imaging is a powerful tool for imaging the orbit, due to the excellent tissue contrast.

Aim of the work: Toevaluate the ability of Diffusion-Weighted MRI in the characterization of the orbital lesions.

Patients and methods: We evaluated 50 patients with 25 malignant and 25 benign orbital lesions. MR examinations were performed with a 1.5-T system. DWIs images were obtained in all patients. The apparent diffusion coefficient (ADC) was calculated and correlated with the pathology results.

Results: The mean ADC value of benign and malignant orbital masses was $1.53 \pm 0.61 \times 10-3 \mathrm{~mm} 2 / \mathrm{s}$ and $0.86 \pm 0.35 \times 10-3 \mathrm{~mm} 2 / \mathrm{s}$, respectively. There was a significant difference in the mean ADC value between benign and malignant orbital masses $(\mathrm{P}<0.001)$. The mean ADC value of benign was significantly higher than that of malignant orbital masses, with considering ADC value of $0.90 \times 10-3 \mathrm{~mm} 2 / \mathrm{sec}$ is a cut off between benign \& malignant orbital masses by sensitivity $76 \%$ and specificity $96 \%$.

Conclusion: We can conclude that the combination of conventional MRI finding and ADC calculation together with clinical finding could improve the diagnostic efficacy of MR imaging in the differentiation between benign and malignant orbital masses with considering an ADC cut off value of $0.90 \times 10-3 \mathrm{~mm} 2 / \mathrm{s}$ between benign and malignant orbital masses with specificity $76 \%$ \& sensitivity $96 \%$.
\end{abstract}

Keywords: MRI; Diffusion-weighted images; Orbital masses; Apparent Diffusion Coefficient.

\section{INTRODUCTION}

Magnetic resonance imaging (MRI) may help the diagnosis of orbital lesions, as there are some pathognomonic features for particular masses as for cavernous hemangioma. However, for cases without these pathognomonic characteristics, it remains difficult to deliver a diagnosis based on MR imaging features because there are often unspecific and overlapping imaging findings. Aggravating this situation, rare tumor entities are unexpected and therefore may be misdiagnosed. ${ }^{1}$

MRI has more values over CT as in its tissue contrast, intrinsic flow sensitivity and non-ionizing radiation. MRI techniques, such as diffusionweighted imaging (DWI) and dynamic contrast enhanced (DCE) MRI, as well as other functional imaging modalities have been showing great potential in oncologic applications of head and neck tumors ${ }^{2}$.

Published data indicate that the use of advanced MRI sequence techniques like DWIs with quantitative apparent diffusion coefficient (ADC) mapping and DCE may provide additional information about the entity of orbital masses. ${ }^{1}$

Generally, due to the enlarged nuclei, hypercellularity, and subsequent limited extra-cellular space, malignant orbital tumors demonstrate lower ADC value than the benign tumors. However, the reproducibility of ADC measurements is still a challenge during clinical practice. Previous study has shown that ADC value can be affected by several factors, such as the magnetic field strength, MRI acquisition parameters, and also the region of interest (ROI) selection methods. ${ }^{2}$ 
This study aims for the assessment of the capability of diffusion-weighted magnetic resonance imaging to differentiate benign from malignant orbital lesions.

\section{PATIENTS AND METHODS}

\section{Study Population}

This study was compliant with our institution committee ethics with a written consent taken from all patients. This study included 50 patients ( 25 male $\& 25$ female), their ages range from ( 4 months to 72 years). All the patient presented to our hospital from june 2017 till September 2019. All the patients were referred from outpatient clinics with orbital lesions to radiology department.

The inclusion criteria was patients of any age group with clinical and radiological findings of orbital masses. The exclsuion criteria was patients with contraindications to MRI or either DW or DCE MRI was not used. Patients with absolute contraindications should not be examined. Relative contraindications may pose a relative hazard, and the type and location of an implant should be assessed prior to the MRI examination.

\section{Patient preparation:}

For the patient who need anathesthia, fasting four hoursbefore the scan is required.

\section{Technique:-}

Conventional and CEMRI: All the patient were evaluated by MRI orbit with diffusion in National cancer Institute, Cairo university, using a $1.5 \mathrm{~T}$ scanner (Achieva, Philips Medical Systems, Best, Netherlands, Release 2.6, and Level 3). All the cases were examined in supine position with standard circularly polarized head coil using the following sequences; Scout T1 TFE (15/5.2 ms) TR/TE, Axial T2WI brain spin echo $(4126 / 100 \mathrm{~ms}) \mathrm{TR} / \mathrm{TE}$, Axial T1 W Ispin echo orbit ( $406 / 15 \mathrm{~ms}$ ) TR/TE, Axial STIRorbit ( $3257 / 20 \mathrm{~ms}$ ) TR/TE, Coronal T1WI spin echo (447/15 ms) TR/TE, Coronal T2W Ispin echo (4417 /100 ms) TR/TE.

After intravenous administration of GadoliniumDTPA $(0.3 \mathrm{mg} / \mathrm{kg})$, contrast enhanced T1WI in axial, sagittal and coronal planes are obtained; T1 W I in axial (406/15 ms) TR/TE, T1WI in sagittal (515/514 ms) TR/TE, T1 WI in coronal (447/15 ms) TR/TE.

MR Diffusion Imaging: All cases were evaluated by DWI in axial planes with ADC map. Diffusionweighted MR imaging was obtained using a multi section single-shot echo planar imaging sequence (TR/TE/NEX: 2200/139 ms/1) field of view $195 \mathrm{~mm}$, matrix size, $128-128$, section thickness $4 \mathrm{~mm}$, section gap $0.3 \mathrm{~mm}$ with $\mathrm{b}$ values of 0 and 800 $\mathrm{s} / \mathrm{mm} 2$.

Post-processing: The ADC maps were calculated automatically by the MRI software. The ADC values were expressed $10^{-3} \mathrm{~mm}^{2} / \mathrm{s}$. The ADC values were measured by manually placing regions of interest in tumor regions on the ADC map. In the solid lesions, regions of interest were placed at the site of enhancing parts on contrast-enhanced $\mathrm{T} 1$ - weighted MR images (and avoiding the cystic, necrotic, and hemorrhagic tumor areas). To calculate the ADC values, the sizes of the regions of interest were adjusted according to the lesion size.

\section{Statistical Analysis}

The statistical analysis of data was done by using Excel and the SPSS program (St atistical Package for Social Science version 25 [IBM Corp., Armonk, NY, USA]). The description of data was done in the form of mean, standard deviation, median, minimum and maximum in quantitative data and using frequency (count) and relative frequency (percentage) for categorical data. The Kolmogorov-Smirnov (K-S) test was done for diagnosis normality of data distribution.The analysis of data was done to test statistical significant difference. Comparisons between quantitative variables were done using the non-parametric Mann-Whitney test. For comparing categorical data, Chi square $(\chi 2)$ test was performed. Exact test was used instead when the expected frequency is less than 5.ROC curve was constructed with area under curve analysis performed to detect best cutoff value of ADC for detection of malignancy. P-values less than 0.05 were considered as statistically significant.

\section{RESULTS}

The study sample was comprised of 50 patients ( 25 female and 25 male) with orbital masses. Patients' age ranged from 4 months to 72 years. The mean age of the cases was 27.04 years \pm 22.93 years (figure 1 ).

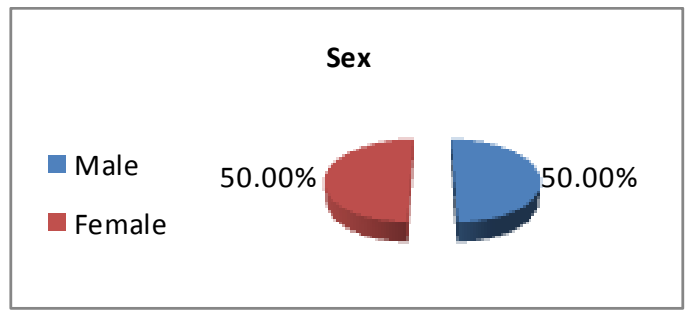

Fig. 1: Sex distribution of our patients.

Conventional MRI criteria of all lesions: On basis of signal intensity on T $1 \& \mathrm{~T} 2$ and contrast uptake, the lesions are distributed as in table (1):

From table (1), most of the lesions (benign and malignant) types showed hypointense S.I (58\%) on T1WIs and hyperintense on T2WIs (44\%). Most of the benign lesions showed hypointense S.I on T 1 WIs and iso/hyperintense on T2WIs. Most of the malignant lesions showed hypointense S.I on T $1 \mathrm{WIs}$ and hyperintense on T2W Is. 


\begin{tabular}{|l|c|c|c|c|c|c|c|c|c|c|c|c|c|}
\hline & \multicolumn{4}{|c|}{ All Lesions } & \multicolumn{4}{c|}{ Benign Lesions } & \multicolumn{3}{c|}{ Malignant Lesions } \\
\hline & \multicolumn{2}{|c|}{ T1 } & \multicolumn{2}{|c|}{ T2 } & \multicolumn{2}{|c|}{ T1 } & \multicolumn{2}{|c|}{ T2 } & \multicolumn{3}{|c|}{ T1 } & \multicolumn{1}{|c|}{ T2 } \\
\hline & $\boldsymbol{f}$ & $\mathbf{\%}$ & $\boldsymbol{f}$ & $\mathbf{\%}$ & $\boldsymbol{f}$ & $\mathbf{\%}$ & $\boldsymbol{f}$ & $\mathbf{\%}$ & $\boldsymbol{f}$ & $\%$ & $\boldsymbol{f}$ & $\boldsymbol{\%}$ \\
\hline Hyper intense & 5 & 10 & 22 & $\mathbf{4 4}$ & 2 & 8 & 8 & 32 & 3 & 12 & 14 & $\mathbf{5 6}$ \\
\hline Hypo intense & 29 & $\mathbf{5 8}$ & 9 & 18 & 14 & $\mathbf{5 6}$ & 6 & 24 & 15 & $\mathbf{6 0}$ & 3 & 12 \\
\hline Iso intense & 13 & 26 & 15 & 30 & 7 & 28 & 8 & $\mathbf{3 2}$ & 6 & 24 & 7 & 28 \\
\hline Mixed & 3 & 6 & 4 & 8 & 2 & 8 & 2 & 8 & 1 & 4 & 1 & 4 \\
\hline Total & 50 & 100 & 50 & 100 & 25 & 100 & 25 & 100 & 25 & 100.0 & 25 & 100.0 \\
\hline
\end{tabular}

Table 1: Frequency $(f)$ distribution of signal intensities by MRI among orbital lesions.

Regarding the enhancement pattern in our results, (40 $\%)$ of benign lesions showed homogenous enhancement and $(28 \%)$ showed heterogeneous enhancement (table 2); however, (60\%) of malignant lesions showed heterogeneous enhancement, and (40 $\%$ ) showed homogenous enhancement (table 2).

\begin{tabular}{|l|c|c|c|c|}
\hline Contrast & $\begin{array}{c}\text { Frequency } \\
\text { of benign } \\
\text { lesions }\end{array}$ & $\boldsymbol{\%}$ & $\begin{array}{c}\text { Frequency } \\
\text { of } \\
\text { malignant } \\
\text { lesions }\end{array}$ & $\%$ \\
\hline No contrast & 8 & 32 & 0 & 0 \\
\hline Homogenous & 10 & $\mathbf{4 0}$ & 10 & 40 \\
\hline Heterogeneous & 7 & 28 & 15 & $\mathbf{6 0}$ \\
\hline Total & 25 & 100 & 25 & 100 \\
\hline
\end{tabular}

Table 2: Frequency distribution of patterns of contrast enhancement among the benign lesions.

Most of the benign lesions (21/25) showed facilitated diffusion.Of the benign lesions, meningioma, invasive fungal sinusitis and dermoid cysts (two cases) showed restricted diffusion on DWIs.

\begin{tabular}{|l|c|c|c|c|}
\hline $\begin{array}{l}\text { Tumor } \\
\text { type }\end{array}$ & $\mathbf{n}$ & $\begin{array}{l}\text { ADC(mean } \\
\mathbf{\& S D}) \\
\times \mathbf{1 0}^{-3} \mathbf{~ m m}^{2} / \mathbf{s}\end{array}$ & Median & $\begin{array}{l}\text { Min }- \text { Max } \\
\times \mathbf{1 0}^{-3} \mathbf{m m}^{\mathbf{2}} \mathbf{s}\end{array}$ \\
\hline Benign & 25 & $1.53 \pm 0.61$ & 1.4 & $0.58-2.80$ \\
\hline Malignant & 25 & $0.86 \pm 0.35$ & 0.8 & $0.35-2.30$ \\
\hline All cases & 50 & $1.19 \pm 0.60$ & 1 & $0.35-2.80$ \\
\hline
\end{tabular}

Table 3: Calculated ADC Values

The calculated ADC values of the lesions ranged from $0.35-2.80 \times 10^{-3} \mathrm{~mm}^{2} / \mathrm{sec}$. The mean, median and range of the ADC values of all patients are shown in table (3). There were statistically significant differences in ADC values between the malignant tumors and benign lesions $(\mathrm{p}<0.001)$.

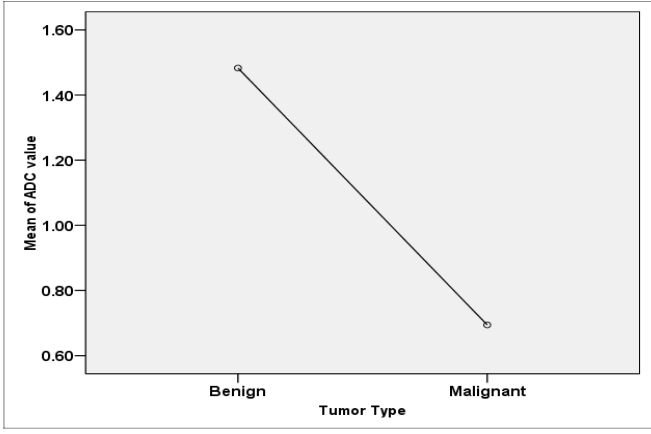

Fig. 2: Mean of ADC value of benign and malignant lesions.

Therefore, ADC value of $0.90 \times 10^{-3} \mathrm{~mm}^{2} / \mathrm{sec}$ is a cut off between benign \& malignant orbital masses by sensitivity $76 \%$ and specificity $96 \%$. Below the cut off value $\left(0.90 \times 10^{-3} \mathrm{~mm}^{2} / \mathrm{sec}\right), 1$ benign \& 20 malignant lesions were found, and above the cut off value 24 benign \& 5 malignant lesions were detected (figure 3).

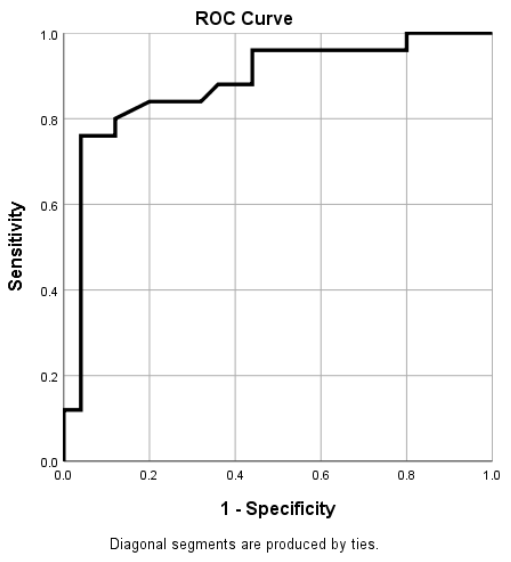

Fig. 3: Receiver operating characteristic (ROC) curve of the ADC value used for differentiating malignant tumors from benign lesions. The area under the curve is 0.882 , which indicates that the accuracy of the test is $86 \%$. 


\section{DISCUSSION}

Our results showed no sex predilection among orbital lesions. This is similar to Xian et al. ${ }^{3}$, Otulana et al. ${ }^{4}$ and Shinder et al. ${ }^{5}$ study results.

Our results showed mean age of the cases 27.04 years (4 months to 72 years). This is close to Gokharman and Aydin ${ }^{6}$ study results that showed mean age of 31 years ( $0-65$ years).

There was no significant correlation between age of patients and differentiation of lesions into benign and malignant lesions as any age may be affected by benign or malignant lesions. Also, Xian et al. ${ }^{\mathbf{3}}$ and Akter et al. ${ }^{7}$ did not found correlation between age and nature of lesions.

In the present study, retinoblastoma $\&$ rhabdomyosarcoma were seen in children. This is similar to Bienvenido and Lawrence ${ }^{\mathbf{8}}$ who mentioned that in pediatrics, the most common orbital malignancy is rhabdomyosarcoma and the most common intraocular malignant lesion is retinoblastoma. Also, similar previous studies as Chung et al. ${ }^{\mathbf{}}$, Jayanta e al. ${ }^{10}$ \& Kralik et al. ${ }^{11}$ who mentioned that rhabdomyosarcoma is the most common extraocular orbital tumor in children.

The current study revealed no significant correlation of the side involvement of lesions and the benignity versus malignancy; this is similar to Sepahdari et al. ${ }^{5}$ and Akter et al. ${ }^{7}$ finding.

Most of the lesions (benign and malignant) types showed hypointense S.I (58\%) on T1WIs and hyperintense on T2WIs (44\%). This is similar to Sultan et al. ${ }^{12}$ study results. In addition, most of the benign lesions showed hypointense S.I on T 1 WIs and iso/hyperintense on T2WIs and most of the malignant lesions showed hypointense S.I on T 1 WIs and hyperintense on T2WIs. This is similar to Akter et al. ${ }^{7}$ study results who mentioned that most of the malignant tumors had low signal intensity on T1WI, iso or slightly high signal intensity on T2WIs and most of the benign tumors had low intensity on T 1 WIs, high or slightly signal intensity on T2WIs.

In the present study, signal intensity with insignificant $P$-value in differentiating between benign and malignant lesions. This is similar to Sepahdari et al. ${ }^{5}$ findings who mentioned that conventional imaging features such as signal intensity on T2WI have not accurately predicted malignancy, and many benign lesions have been sampled for biopsy to exclude clinically suspected malignancy.

In addition, Sepahdari et al. ${ }^{\mathbf{1 3}}$ mentioned that although T1-, T2-, and contrast-enhanced T1weighted sequences can often distinguish various ocular neoplasms from simulating lesions, certain diagnostic dilemmas remain.

However, there was disagreement with other studies like Xiao-Quan et al. ${ }^{2}$ and Xian et al. ${ }^{3}$ studies who mentioned isointense signal on T2WI was found to be a predictive feature for orbital malignancy in their studies. This was explained by that most common primary orbital malignant tumors in in their studies is lymphoma, which usually manifests as iso-intense on T2WI compared to extraocular muscles, so it is not surprising that iso-intense on T2WI could be an important predictive factor for malignancy.

In our study, most of the benign lesions showed homogenous enhancement. This is similar to Akter et al. ${ }^{7}$ and Sultan et al. ${ }^{\mathbf{1 2}}$ study results. Also, most of malignant lesions showed heterogeneous enhancement, this in keeping with Akter et al. ${ }^{7}$ who mentioned that most of the malignant tumors show heterogeneous enhancement.

In our study, most of the benign lesions showed facilitated diffusion and most of the malignant lesions showed restricted diffusion on DWIs. This was similar to Roshdy et al. ${ }^{\mathbf{1 4}}$ and Sepahdari et al. ${ }^{\mathbf{5}}$ study results.

In addition, our study results showed a case of optic nerve meningioma that showed restricted diffusion, this was similar to Sultan et al. ${ }^{\mathbf{1 2}}$ study results that showed that optic nerve meningioma shows restricted diffusion because of their hypercellular nature.

In the present study, a case of invasive fungal sinusitis showed restricted diffusion, this was similar to Safder et al. ${ }^{15}$ study results which were explained by ischemia $\&$ infarction.

Also, we had one case of malignant peripheral nerve sheath tumor that showed facilitated diffusion, this was similar to Abdelrazek et al. ${ }^{\mathbf{1 6}}$ study results as malignant peripheral nerve sheath tumor revealed a high ADC value due to the presence of a prominent mxyoid component within the tumor.

In the present study, one case of lymphoma \& mucoepidermoid carcinoma, two cases of squamous cell carcinomas, retinoblastoma \& intraocular melanoma, three cases of metastasis \& rhabdomyosarcoma showed restricted diffusion, this was similar to Sultan et al. ${ }^{\mathbf{1 2}}$ study results.

In the present study, two cases of adenoid cystic carcinoma of the lacrimal gland showed restricted diffusion, this was similar to Elkhamary ${ }^{17}$ study results.

Our results revealed that the mean ADC value of benign and malignant orbital masses was $1.53 \pm 0.61$ $\mathrm{x} 10^{-3} \mathrm{~mm}^{2} / \mathrm{s}$ and $0.86 \pm 0.35 \times 10^{-3} \mathrm{~mm}^{2} / \mathrm{s}$, respectively.

Similar, Zareen et al. ${ }^{\mathbf{1 8}}$ who reported a mean ADC value of $0.77 \times 10-3 \mathrm{~mm}^{2} / \mathrm{s}$ for malignant lesions and a mean ADC for benign lesions equals $1.23 \times 10$ $3 \mathrm{~mm}^{2} / \mathrm{s}$. Sepahdari et al. ${ }^{5}$ reported a mean ADC value of $1.43 \pm 0.41 \times 10-3 \mathrm{~mm}^{2} / \mathrm{s}$ for malignant lesions and a mean ADC for benign lesions of $0.90 \pm$ 
$0.36 \times 10-3 \mathrm{~mm}^{2} / \mathrm{s}$. Esam ${ }^{19}$ reported that a mean ADC value for malignant masses was $0.77 \pm 0.22 \times 10^{-3} \mathrm{~mm}^{2} / \mathrm{s}$ and for benign lesions was $1.51 \pm 0.39 \times 10^{-3} \mathrm{~mm}^{2} / \mathrm{s}$. Sepahdari et al. ${ }^{\mathbf{1 3}}$ reported that the mean ADC value for malignant masses was $1.02 \pm 0.42 \times 10^{-3} \mathrm{~mm}^{2} / \mathrm{s}$, and for benign lesions, it was $1.36 \pm 0.41 \times 10^{-3} \mathrm{~mm}^{2} / \mathrm{s}$. Abdelrazek et al. ${ }^{16}$ reported that the mean $\mathrm{ADC}$ value for malignant masses was $0.8 \pm 0.34 \times 10^{-3} \mathrm{~mm}^{2} / \mathrm{s}$, and for benign lesions it was $1.53 \pm 0.35 \times 10^{-3} \mathrm{~mm}^{2} / \mathrm{s}$. The malignant group demonstrated significantly lower ADC values $(0.7110 .260$ versus 1.1870 .389 $\times 10^{-3} \mathrm{~mm}^{2} / \mathrm{s}^{2}$.

There was a significant difference between ADC value of benign and malignant lesions $(P<0.001)$. ROC analysis was used to detect the cutoff point differentiating malignant lesions from benign pathologies. The area under the curve was larger than $0.882(P<0.001)$. When $0.90 \times 10^{-3} \mathrm{~mm}^{2} / \mathrm{s}$ was used as the cutoff ADC to distinguish between benign and malignant lesions, sensitivity was calculated as $76 \%$ and specificity as $96 \%$.

The outcomes of the current study showed clear differences between malignant and benign lesions. This was in agreement with similar previous studies as Sepahdari et al. ${ }^{\mathbf{5}}$ and Esam. ${ }^{19}$

In our study, a threshold value of $0.9 \times 10^{-3} \mathrm{~mm}^{2} / \mathrm{sec}$, ADC was $76 \%$ sensitive (19 of 25), $96 \%$ specific ( 24 of 25 ), and $86 \%$ accurate (43 of 50 ) for differentiating malignant from benign lesions.

We concluded that the sensitivity of ADC in differentiating benign from malignant orbital masses in our study was $76 \%$ indicating a high true positive rate. Hence, if the average ADC is below $0.90 \times 10$ ${ }^{3} \mathrm{~mm}^{2} / \mathrm{sec}$, there is high probability that the mass will be malignant with high specificity $96 \%$. Hence, the ADC value can be considered a biomarker to differentiate malignant and benign tumors

In the present study, optic nerve glioma $(n=4)$ show $\mathrm{ADC}$ range of $\left(1.0-2.50 \times 10^{-3} \mathrm{~mm}^{2} / \mathrm{s}\right)$. This is attributed to their low cellularity and low proliferative indices. This was in agreement with Nickerson et al. ${ }^{20}$ who use of DWI and dynamic contrast-enhanced imaging to calculate the diffusivity and permeability of optic nerve glioma. Similar to other pilocytic astrocytomas, it showed ADC values in the range of $1.2-2.09 \times 10^{-3} \mathrm{~mm}^{2} / \mathrm{s}$ Sultan et al. 12

Our study showed no significant difference between the mean ADC value of optic nerve glioma $\left(1.39 \pm 0.75 \times 10^{-3} \mathrm{~mm}^{2} / \mathrm{s}\right)$ and optic nerve meningioma $\left(1.04 \times 10^{-3} \mathrm{~mm}^{2} / \mathrm{s}\right)(\mathrm{P}=0.56)$. Overlap is seen between ADC values for cases of optic nerve glioma and meningioma, this is similar to Esam ${ }^{19}$ results.

In our study, two ocular melanomas showed restricted diffusion with a mean ADC value of
$0.81 \times 10^{-3} \mathrm{~mm}^{2} / \mathrm{s}$. This is close to Erb-Eigner et al. ${ }^{\mathbf{2 2}}$ who reported that ocular melanomas show marked restricted diffusion with a mean ADC value of $0.891 \times 10^{-3} \mathrm{~mm}^{2} / \mathrm{s}$.

In our study, we had one case of atypical meningioma showed restricted diffusion with ADC value of $0.70 \times 10^{-3} \mathrm{~mm}^{2} / \mathrm{s}$. This is similar to Raymond et al. ${ }^{23}$ who reported that an ADC cutoff value of $0.70 \times 10^{-3} \mathrm{~mm}^{2} /$ s provided a sensitivity for diagnosing atypical/aggressive meningiomas of $29 \%$, specificity of $94 \%$, positive predictive value of $67 \%$, and negative predictive value of $75 \%$.

\section{AKNO WELDGMENT}

My deep appreciation and gratitude to Prof. Dr. Mohammad Farouk Aggag professor of Radiodiagnosis, Faculty of medicine, Al-Azhar University, and Prof. Dr. Abdullah Elhusseiny Abdelgawwad, professor of Ophthalmology, Faculty of medicine, Al-Azhar University, for their remarkable effort, great experience had added much to my knowledge, their great support and kind close supervision .

I would like to express my deep thanks and sincere gratitude to Prof. Dr. Wafik Ebrahim Aly, Asst. professor of Radiodiagnosis, Faculty of medicine, Al-Azhar University and Dr. Ayda Aly Youssef, lecturer of Radiodiagnosis, National Cancer Institute, Cairo University for their valuable advice, helpful suggestions and kind supervision.

\section{CONCLUSION}

So we can conclude that the combination of conventional MRI findingand ADC calculationtogether with clinical finding could improve the diagnostic efficacy of MR imaging in the differentiation between benign and malignant orbital masses with considering a cut off ADC value of $0.90 \times 10^{-3} \mathrm{~mm}^{2} / \mathrm{s}$ between benign and malignant orbital masses with specificity $76 \%$ \& sensitivity $96 \%$.

\section{REFERENCES}

1. Sa-Ra R, Patrick A, Eberhard S. et al. "Characterization of Orbital Masses by Multiparametric MRI."NeuroImage. European Journal of Radiology.2015;85(2): $324-336$

2. Xiao-Quan $\mathrm{X}$, Hao H, Guo-Yi S. et al. Diffusion Weighted Imaging for Differentiating Benign from Malignant Orbital T umors: Diagnostic Performance of the Apparent Diffusion Coefficient Based on Region of Interest Selection Method. Korean J Radiol. 2016;17(5):650-656.

3. Xian J, Zhengyu Z, Zhenchang W. et al. Value of MR Imaging in the Differentiation

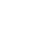


of Benign and Malignant Orbital Tumors in Adults. European Radiology, SpringerVerlag, EurRadiol.2010; 20(7): 1692-1702.

4. Otulana TO, Sogebi OA, Ajibode HA. et al. Etiological pattern, clinical presentation, and management challenges of proptosis in a tertiary hospital in South West Nigeria. Niger J Gen Pract. 2016;(14) 2:28-32.

5. Sepahdari AR, Aakalu VK, Setabutr P. et al. Indeterminate Orbital Masses: Restricted Diffusion at MR Imaging with Echo-planar Diffusion-weighted Imaging Predicts Malignancy. Radiology. 2010;256:554-564.

6. Gokharman D and Aydin S. Magnetic Resonance Imaging in Orbital Pathologies: A Pictorial Review. Journal of the Belgian Society of Radiology. 2018; 102(1): 1-8.

7. Akter GS, Hasan MZ, Sana DS. et al. Valuation of Four-Quadrant Location Method in Diagnosis and Differential Diagnosis of the Orbital Tumor by Comparison Study Combining CT and MRI with Pathology. $J$ Pain Relief. 2017; 6: 306-9.

8. Bienvenido VC and Lawrence K. Pediatric tumors of the eye and orbit. Pediatr Clin North Am. 2003; 50(1): 149-172.

9. Chung EM, Murphey MD, Specht CS. et al. From the archives of the AFIP: pediatric orbit tumors and tumor like lesions: neuroepithelial lesions of the ocular globe and optic nerve. Radiographics. 2007;27(4) :1159-1186.

10. Jayanta KD, Tiwary BK, Paul SB. et al. Primary orbital rhabdomyosarcoma with skeletal muscle metastasis. Oman $J$ Ophthalmol. 2010; 3(2): 91-93.

11. Kralik SF, Kathryn MH, Remy RL. et al. Orbital Infantile Hemangioma and Rhabdomyosarcoma in Children: Differentiation Using Diffusion-weighted Magnetic Resonance Imaging. American Association for Pediatric Ophthalmology and Strabismus. 2018;22(1):27-31.

12. Sultan AA, Hanyal-Backry M, Alhefney EM. et al. Role of MR Spectroscopy and Diffusion-weighted Imaging in Diagnosis of Orbital Masses. The Egyptian Journal of Radiology and Nuclear Medicine. 2018; 49(1): 45-53.

13. Sepahdari AR, Politi LS, Aakalu VK. et al. Diffusion-weighted imaging of orbital masses: multi-institutional data support a 2ADC threshold model to categorize lesions as benign, malignant, or indeterminate. AJNR Am J Neuroradiol. 2014; (35): 170-175.

14. Roshdy N, Shahin M, Kishk H. et al. MRI in diagnosis of orbital masses. Curr Eye Res. 2010;35(11):986-991.

15. Safder S, Carpenter JS, Roberts TD. et al. The "Black Turbinate" Sign: An Early MR Imaging Finding of Nasal Mucormycosis. American Journal of Neuroradiology. 2009; 31(4):771-4.

16. Abdel Razek AA, Elkhamary S and Mousa A. Differentiation between benign and malignant orbital tumors at 3-T diffusion MR-imaging. Springer-verlag Neuroradiology. 2011;53:517-522.

17. Elkhamary SM. Lacrimal gland lesions: Can addition of diffusion-weighted MR imaging improve diagnostic accuracy in characterization?. The Egyptian Journal of Radiology and Nuclear Medicine. 2012; 43: 165-172.

18. Zareen $\mathrm{F}$, Ichikawa $\mathrm{T}$, Ishigame $\mathrm{K}$. et al. Orbital Masses: the Usefulness of DiffusionWeighted Imaging in Lesion Categorization. ClinNeuroradiol. 2014; 24(2):129-34.

19. Esam MH. Characterization of orbital masses by diffusion-weighted Magnetic Resonance Imaging (DWI) and Apparent Diffusion Coefficient (ADC) value. The Egyptian Journal of Radiology and Nuclear Medicine. 2017; 48(1): 115-123.

20. Nickerson JP, Salmela MB, Koski CJ. et al. Diffusion tensor imaging of the pediatric optic nerve: intrinsic and extrinsic pathology compared to normal controls. Magn Reson Imaging J. 2010; 32: 76-81.

21. Kapur R, Mafee MF, Lamba R. et al. Orbital schwannoma and neurofibroma: role of imaging. Neuroimaging Clin $N \mathrm{Am}$. 2005;15:159-174.

22. Erb-Eigner K, Willerding G, T aupitz M. et al Diffusion-Weighted Imaging of Ocular Melanoma. Invest Rad iol. 2013; 48:702-707.

23. Raymond YH, Wenya LB, Brent G. et al. Imaging and diagnostic advances for intracranial meningiomas. Neuro-Oncology. 2019; 21(1): 44-61 